\title{
Registros notables del puerco espín tropical Sphiggurus mexicanus (Erethizontidae) en el estado de Oaxaca, México
}

\author{
Ma. Emma Cisneros-Palacios ${ }^{1}$, Gladys Reyes-Macedo ${ }^{2}$, Ángel Méndez ${ }^{3}$, Gabriela Monroy y Carlos Ramírez Calderón ${ }^{5}$ \\ ${ }^{1}$ Instituto Politécnico Nacional. Departamento de Investigación, Centro Interdisciplinario de Investigación para el Desarrollo \\ Integral Regional Unidad Oaxaca. Calle Hornos 1003, Santa Cruz Xoxocotlán 71230, Oaxaca. México. E-mail: emmacisneros3@ \\ hotmail.com (MECP) \\ ${ }^{2}$ Vinculación Interdisciplinaria para el Desarrollo Ambiental y lo Social, A. C. VIDAS A. C. Calle Zaa, Casa 102, Dpto. 201, Oaxaca de \\ Juárez 68143. Oaxaca, México. E-mail: greyesmacedo@yahoo.com.mx (GRM) \\ ${ }^{3}$ Departamento de Monitoreo Biológico, Conservación Biológica y Desarrollo Social, A. C., Aguayo 3, Despacho A, Del Carmen \\ Coyoacán 04100. Distrito Federal, México. E-mail: angelmendez@conbiodes.com (AM) \\ ${ }^{4}$ Laboratorio de Sistemas de Información Geográfica, Departamento de Zoología, Instituto de Biología, Universidad Nacional \\ Autónoma de México. Circuito Exterior s/n, Ciudad Universitaria, Coyoacán 04510. Distrito Federal, México. E-mail: beu_ribetzin@ \\ hotmail.com (GM) \\ ${ }^{5}$ Domicilio conocido, Concepción Pápalo, Cuicatlán. Oaxaca, México. E-mail: comisariado_19@hotmail.com (CRC) \\ ${ }^{*}$ Corresponding author
}

Introduction: The tropical porcupine, Sphiggurus mexicanus is distributed from west Panamá and Costa Rica to Michoacán on the Pacific versant and San Luis Potosí on the Gulf of Mexico basin. It dwells in tropical and semitropical dry forests, at altitudes from 0 to 3,200 m; it was recently recorded at temperate pine and pine-oak forests in Michoacán. In Oaxaca this species had been recorded only at Gulf of Mexico coastal plain, Pacific coastal plain and Isthmus of Tehuantepec, at low elevations from 0 to $500 \mathrm{~m}$, and inhabiting costal dunes, tropical evergreen and deciduous forests, grasslands, farmland and secondary vegetation. The goal of this paper is to report new data on the distribution of the tropical porcupine in Oaxaca, México.

Methods: Five records are based on a voucher specimen and direct sight of individuals or remains found along field trips. A sixth record was obtained with Camera traps.

Results: We report a specimen of S. mexicanus found dead on the road near Colonia Cuauhtémoc, this record lies within the known distribution for the species. We also report five noteworthy records for the Mexican tree porcupine, three at Sierra Norte and two at Cuicatlán- Tehuacán Valley regions, all within cloud forests, at altitudes from 1,673 to $2,668 \mathrm{~m}$.

Discussion and conclusions: The three records at Sierra Norte expand the geographical range for this species $34 \mathrm{~km}$ to the southwest, from San Juan Petlapa, the closest known locality. These records along with the two from Cuicatlán valley are the first records for the tropical porcupine within cloud forests in Oaxaca and at higher altitudes than those previously reported. The record of a small immature female implies that the species is actually breeding within this type of forest in Oaxaca. These five records are noteworthy as they expand the known geographical and ecological distribution of the Mexican tree porcupine in Oaxaca. Distributional data are fundamental to the species biology and critical to conservation strategies.

Key words: cloud forest; Cuicatlán- Tehuacán valley; mammals; Oaxaca; Sierra norte.

Introducción: El puerco espín tropical, Sphiggurus mexicanus, se distribuye desde el oeste de Panamá y Costa Rica hasta Michoacán, por la vertiente del Pacífico, y hasta San Luis Potosí, por la vertiente del Golfo de México. Habita bosques tropicales y subtropicales secos, de 0 a 3,200 m. Recientemente, se registró en bosques templados de pino y pino encino en Michoacán. En Oaxaca, esta especie se ha registrado solo en las regiones de la planicie costera de Golfo, en la planicie costera del Pacífico y en el Istmo de Tehuantepec, de los 0 a los $500 \mathrm{~m}$ de altitud, en vegetación de dunas costeras, bosque tropical perennifolio y caducifolio, pastizales, cultivos y vegetación secundaria. El objetivo del presente trabajo es aportar nuevos datos sobre la distribución del puerco espín tropical en Oaxaca, México. 
Métodos: Cinco registros están basados en un ejemplar colectado y en la observación directa de individuos o de restos encontrados durante recorridos en campo. El sexto es un registro con fototrampa.

Resultados: Se encontró un ejemplar muerto de Sphiggurus mexicanus sobre la terracería cerca de la Colonia Cuauhtémoc, este concuerda con la distribución conocida para la especie. También se reportan cinco registros notables del puerco espín tropical: tres en la región Sierra Norte y dos en el valle de Cuicatlán-Tehuacán, todos ellos dentro de bosque mesófilo, entre 1,673 y 2,668 m de altitud.

Discusión y conclusiones: Los tres registros en Sierra Norte expanden la distribución geográfica de esta especie $34 \mathrm{~km}$ al suroeste de San Juan Petlapa, la localidad conocida más cercana. Estos registros, junto con los dos del Valle de Cuicatlán, son los primeros del puerco espín tropical dentro del bosque mesófilo en Oaxaca y a altitudes mayores que las reportadas anteriormente. El registro de una cría hembra indica que la especie se reproduce en este tipo de vegetación en Oaxaca. Los cinco registros son notables, ya que amplían la distribución geográfica y ecológica de la especie en Oaxaca. Los datos sobre distribución son fundamentales para conocer la biología de la especie y son críticos para cualquier estrategia de conservación.

Palabras clave: Bosque mesófilo, Cuicatlán, mamíferos, Oaxaca, Sierra Norte.

\section{Introducción}

El puerco espín tropical (Sphiggurus mexicanus) se distribuye desde el oeste de Panamá y Costa Rica hasta México (Reid 1997). En este último país, se distribuye por todo el sur y sureste. En Oaxaca, su distribución continúa por ambas vertientes y llega hasta el sureste de Michoacán, por la vertiente del Pacífico (Monterrubio-Rico et al. 2010), y hasta San Luis Potosí, por la vertiente del Golfo de México (Aranda 2000). Habita en los bosques tropicales y subtropicales a altitudes de 0 a 3,200 m y, a bajas elevaciones, parece preferir hábitats estacionalmente secos (Reid 1997). Recientemente se reportó su presencia en los bosques templados de pino y pino encino (a altitudes de 770 a 920 m) del suroeste de Michoacán (Monterrubio-Rico et al. 2010). Los altos índices de pérdida y fragmentación de su hábitat, así como la cacería y el escaso estudio sobre esta especie en particular, no han permitido conocer más ampliamente su ecología y distribución (Lira et al. 2005; López et al. 2009; Lorenzo et al. 2014).

Para el estado de Oaxaca se ha documentado la presencia de S. mexicanus en la región de la planicie costera del Golfo, distrito de Tuxtepec; en la región de la cañada, distrito de Cuicatlán; en la planicie costera del Pacífico, distrito de Jamiltepec, y en el Istmo de Tehuantepec, distritos de Juchitán y Tehuantepec. Los registros anteriores lo sitúan en altitudes entre 0 y $500 \mathrm{~m}$. Ocupa dunas costeras, bosque tropical perennifolio, bosque tropical caducifolio, pastizales, vegetación secundaria y cultivos (Goodwin 1969; Hall 1981; Navarro y Meave 1998; Briones-Salas y SánchezCordero 2004; Lira et al. 2005; CONABIO 2010a; Voss 2011; Lorenzo et al. 2014).

\section{Material y métodos}

Cuatro registros se obtuvieron mediante observación directa de individuos o de sus restos colectados y/o fotografiados durante recorridos en campo, que se hicieron a pie, a diferentes horas durante el día y con diferentes longitudes, como parte de diversos proyectos de investigación sobre vertebrados terrestres, realizados por E. Cisneros del CIIDIR-OAXACA. Durante un recorrido a pie efectuado por el comité de vigilancia ambiental comunitaria, dentro de su área de conservación, se obtuvo un quinto registro mediante la observación directa de los restos de un ejemplar, los cuales fueron fotografiados, y que consisten en huesos y púas.

Otro registro más se obtuvo mediante fototrampeo. Para ello se utilizó una fototrampa marca Ltl Acorn de 12 megapíxeles modelo 5210, que posee un sistema infrarrojo activado por el calor y por el movimiento. Estuvo activa durante 24 horas continuas; se programó con un intervalo de 1 minuto entre evento; cada evento constó de dos fotografías con una resolución de 600 dpi y un video de 30 segundos. La fototrampa se colocó en un árbol de Pinus sp. a una altura aproximada 
de $30 \mathrm{~cm}$. Tras un esfuerzo acumulado de 12 días-trampa, la fotografía fue tomada a las 23:25 horas. Las altitudes y coordenadas reportadas se obtuvieron en campo empleando un GPS.

\section{Resultados}

Reportamos seis registros de S. mexicanus obtenidos entre 1990 y 2012 en diversas localidades de Oaxaca. Cinco de ellos son notables porque amplían la distribución geográfica y ecológica de la especie (Tabla 1; Figura 1).

Cuatro registros son resultado de diversos proyectos de investigación sobre vertebrados terrestres, Ilevados a cabo por investigadores del CIIDIR, Unidad Oaxaca. El primero es un ejemplar encontrado muerto sobre la terracería, $1.6 \mathrm{~km}$ al O de la colonia Cuauhtémoc, en el Istmo de Tehuantepec, en vegetación secundaria y zonas de cultivo (Registro 1; Tabla 1). Aunque éste no sea un registro nuevo en dicha región, contribuye a conocer mejor el intervalo de distribución de esta especie en el estado. El ejemplar se encuentra depositado en la Colección Mastozoológica del CIIDIR Unidad Oaxaca (registro ante el INE: OAX.MA.025.0497), con el número de catálogo OAX.MA-446.

Tres registros notables (Registros 2, 3 y 4; Tabla 1) se localizan en la Sierra Norte de Oaxaca, a $1.4 \mathrm{~km}$ y $1.45 \mathrm{~km}$ al SE de Tanetze de Zaragoza. Una cría hembra fue fotografiada en la localidad de Tarántulas, a $1.3 \mathrm{~km}$ al ESE de San Juan Juquila Vijanos. Se encontró esta cría aproximadamente a $60 \mathrm{~cm}$ de altura sobre una liana gruesa. El ejemplar medía aproximadamente $47 \mathrm{~cm}$ de largo, incluida la cola. No presentaba las púas completamente desarrolladas, ya que no sobresalían del pelaje, como sucede en ejemplares adultos (Figura 1). No pudo localizarse a la madre, aunque se le escuchó responder al llanto de la cría desde la parte alta de los árboles.

El 28 de julio de 2011, en la ranchería La Reforma, a 7.95 km al SSE, en línea recta de San Francisco Chapulapa, perteneciente a la agencia de San Alejo el Progreso, en la región de la cañada de Oaxaca, personal de VIDAS A.C. y del comité de vigilancia ambiental comunitaria encontraron y fotografiaron los restos de un ejemplar de S. mexicanus (Registro 5; Tabla 1). El hallazgo ocurrió durante su recorrido de inspección dentro del área de conservación. Se trata del primer registro de la especie en el bosque mesófilo de la región del Valle de Cuicatlán-Tehuacán. Los restos consisten en el esqueleto incompleto (sin cráneo) y en numerosas púas características de la especie.

El último registro se obtuvo el 20 de julio de 2012, a 6.89 km en línea recta al ENE de Concepción Pápalo, también en vegetación de bosque mesófilo de montaña (Registro 6; Tabla 1), como parte del proyecto "Red de Monitoreo de Fauna Silvestre", que lleva a cabo la asociación "Conservación Biológica y Desarrollo Social" (CONBIODES A. C.), mediante el método de fototrampeo. La fotografía se encuentra en la Colección de Foto colectas Biológicas (CFB) del Instituto de Biología de la Universidad Nacional Autónoma de México, con el número de catálogo: IBUNAM-CFB-13201.

Tabla 1. Nuevos registros de Sphiggurus mexicanus en Oaxaca, México. ${ }^{1}$ Metros sobre el nivel del mar. ${ }^{2}$ Bosque mesófilo de montaña

\begin{tabular}{|c|c|c|c|c|c|}
\hline Registro & Fecha & Latitud/Longitud & Municipio/Distrito & Altitud ${ }^{1}$ & Vegetación \\
\hline 1 (ejemplar) & 05/06/1990 & $17.09896^{\circ} \mathrm{N},-94.9141^{\circ} \mathrm{O}$ & Matías Romero, Juchitán & 98 & secundaria \\
\hline 2 (visual) & $26 / 06 / 1994$ & $17.37175^{\circ} \mathrm{N},-96.28926^{\circ} \mathrm{O}$ & Tanetze de Zaragoza, Villa Alta & 1,673 & $\mathrm{BMM}^{2}$ \\
\hline 3 (visual) & 19/10/1999 & $17.35931^{\circ} \mathrm{N},-96.27658^{\circ} \mathrm{O}$ & Tanetze de Zaragoza, Villa Alta & 1,871 & BMM \\
\hline 4 (fotografía) & $07 / 11 / 2000$ & $17.35201^{\circ} \mathrm{N},-96.29195^{\circ} \mathrm{O}$ & San Juan Juquila Vijanos, Villa Alta & 1,742 & BMM \\
\hline 5 (fotografía) & $28 / 07 / 2011$ & $17.86788^{\circ} \mathrm{N},-96.75801^{\circ} \mathrm{O}$ & San Francisco Chapulapa, Cuicatlán & 2,061 & BMM \\
\hline 6 (fotografía) & $20 / 07 / 2012$ & $17.84841^{\circ} \mathrm{N},-96.81676^{\circ} \mathrm{O}$ & Concepción Pápalo, Cuicatlán & 2,668 & BMM \\
\hline
\end{tabular}



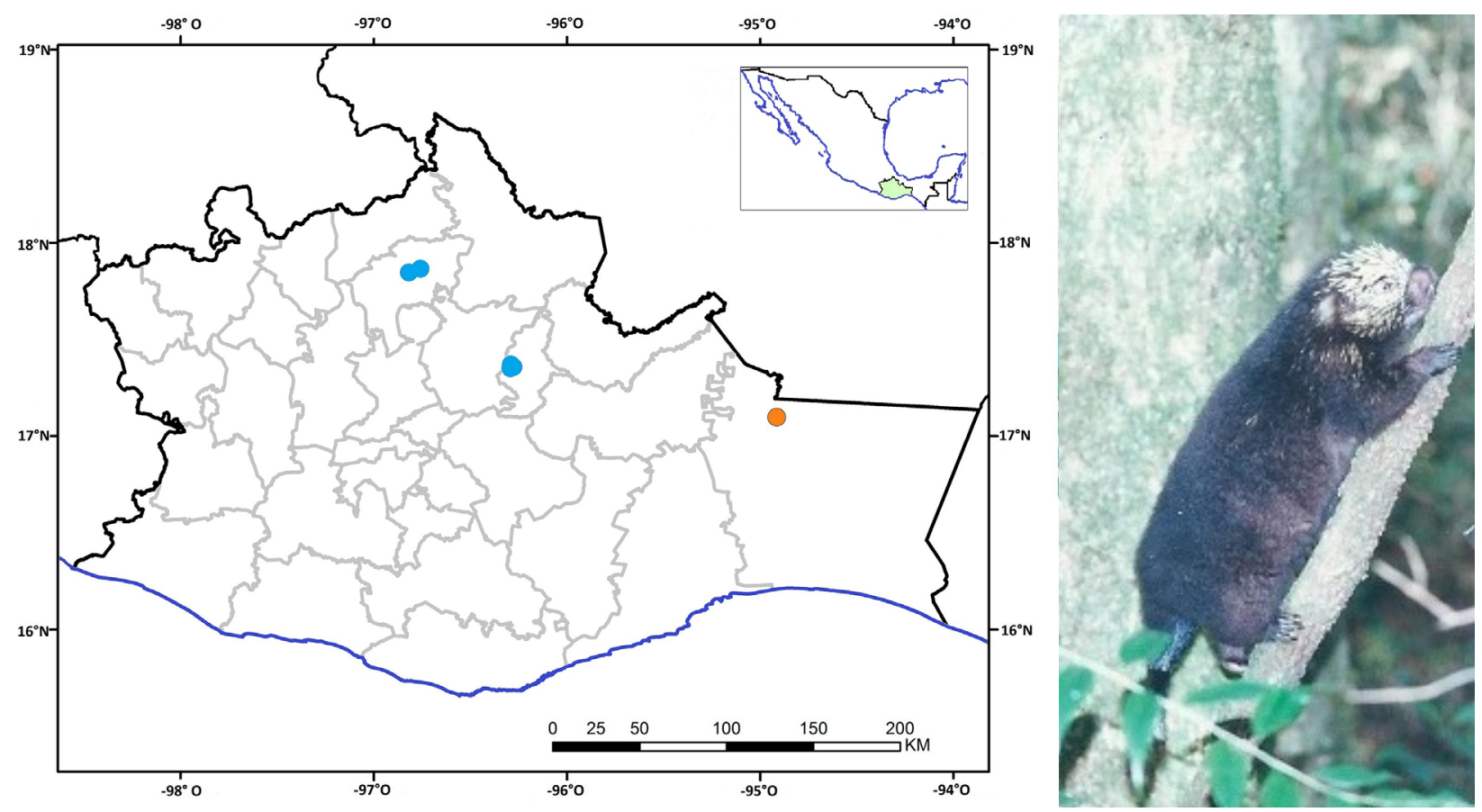

Figura 1. Nuevos registros del puerco espín tropical, Sphiggurus mexicanus en Oaxaca, México. Círculos azules = registros notables; círculo naranja = registro adicional dentro de la distribución conocida para la especie. Cría de puerco espín tropical fotografiada cerca de San Juan Juquila Vijanos, Oaxaca, México (Foto: Emma Cisneros Palacios).

\section{Discusión y conclusiones}

Los presentes registros son los primeros para el distrito de Villa Alta en la Sierra Norte de Oaxaca. En relación con el tipo de vegetación ocupado por la especie, cinco de los seis registros se ubican dentro del bosque mesófilo de montaña en el estado. En particular, el registro de una cría en la localidad de Tarántulas implica que la especie se reproduce en dicho tipo de vegetación y no se trata solo de un ejemplar inmaduro en proceso de dispersión. Aunque Briones-Salas y SánchezCordero (2004) indican que S. mexicanus se distribuye en el estado de Oaxaca de los 0 a 500 metros de altitud, cinco de nuestros registros expanden el rango altitudinal de la especie desde 1,673 hasta 2,668 $\mathrm{m}$ en los bosques mesófilos del estado, considerados como los ecosistemas más amenazados en el ámbito nacional (CONABIO 2010b).

Sphiggurus mexicanus es una especie considerada rara. Su biología es poco conocida y se le ubica en la categoría de amenazada, según la NOM-059-SEMARNAT-2010 (SEMARNAT 2010), y en la categoría de preocupación menor (Least concern) de la UICN (Pino et al. 2008), debido a sus hábitos arborícolas y a su dependencia de bosques tropicales húmedos, los cuales están desapareciendo rápidamente (Lorenzo et al. 2014). Los datos aquí presentados contribuyen a la ubicación de poblaciones de la especie en el estado, lo que permitirá realizar estudios que aporten mayor información sobre la biología de esta especie, y resultan críticos para desarrollar estrategias de conservación.

\section{Agradecimientos}

Agradecemos a los integrantes de los Comités de Vigilancia Ambiental Comunitaria de la Ranchería La Reforma y de Concepción Pápalo; a las autoridades agrarias de la Agencia de San Alejo el Progreso, por su interés y apoyo en la generación de conocimiento científico; a M. M. Pablo Cruz por su apoyo en campo, y al personal de VIDAS A. C., por el desarrollo del proyecto en 
San Alejo el Progreso; a F. Botello y E. Villaseñor, responsables del proyecto de Red de Monitoreo de Fauna Silvestre en la Reserva de la Biosfera Tehuacán-Cuicatlán; a los integrantes del Departamento de Monitoreo Biológico de CONBIODES A.C., quienes apoyaron en la obtención de los registros; a la CONAFOR, por la ayuda financiera para el proyecto de pago por servicios ambientales hidrológicos en San Alejo el Progreso, San Francisco Chapulapa, Oaxaca, y a R. Rivera por la elaboración del mapa.

\section{Literatura citada}

Aranda, M. 2000. Huellas y otros rastros de los mamíferos grandes y medianos de México. Instituto de Ecología, A. C. Xalapa, México.

Briones-Salas, M., y V. Sánchez-Cordero. 2004. Mamíferos. Pp. 423-447 in Biodiversidad de Oaxaca (García-Mendoza, A. J., M. J. Ordoñez, y M. Briones-Salas, eds.). Instituto de Biología, UNAM-Fondo Oaxaqueño para la Conservación de la Naturaleza-World Wild Fund. México.

Conabio (Comisión Nacional para el Conocimiento y Uso de la Biodiversidad). 2010a. Coendou mexicanus (Puerco espín tropical). Distribución conocida. http://www.conabio.gob. $\mathrm{mx} /$ informacion/gis/?vns=gis_root/biodiv/spryp/a_amenz/amamma/arodentia/ coemex_dcgw.

Conabio (Comisión Nacional para el Conocimiento y Uso de la Biodiversidad). 2010b. El bosque mesófilo de montaña en México: Amenazas y oportunidades para su conservación y manejo sostenible. Comisión Nacional para el Conocimiento y Uso de la Biodiversidad. Ciudad de México, México.

Goodwin, G. G. 1969. Mammals from the state of Oaxaca, Mexico, in the American Museum of Natural History. Bulletin of the American Museum of Natural History 141:1-269.

HaLL, R. E. 1981. The Mammals of North America. John Wiley and Sons. New York, EE. UU.

LiRa, T. I., L. Mora, M. A. CAmacho, y R. E. Galindo. 2005. Mastofauna del Cerro de la Tuza, Oaxaca. Revista Mexicana de Mastozoología 9:6-20.

López, J. A., C. Lorenzo, F. Barragán, y J. Bolaños. 2009. Mamíferos terrestres de la zona lagunar del Istmo de Tehuantepec, Oaxaca, México. Revista Mexicana de Biodiversidad 80:491-505.

Lorenzo, C., E. C. Sántiz, D. A. Navarrete, y J. Bolaños. 2014. Causes and consequences of change rates in the habitat of the threatened tropical porcupine, Sphiggurus mexicanus (Rodentia: Erethizontidae) in Oaxaca, México: implications for its conservation. Revista de Biología Tropical 62:481-1494.

Monterrubio-Rico, T., J. M. Ortega-Rodríguez, N. Mendoza-Cárdenas, R. Cansino-Murillo, y A. Pérez-Arteaga. 2010. Distributional and ecological records of the Mexican hairy dwarf porcupine (Sphiggurus mexicanus) from Michoacán, Mexico. The Southwestern Naturalist 55:139-142.

Navarro, S. A., y J. A. Meave. 1998. Inventario de la biodiversidad de vertebrados terrestres de los Chimalapas, Oaxaca. Universidad Nacional Autónoma de México. Facultad de Ciencias. Informe final SNIB-CONABIO proyecto No B002, Ciudad de México, México. Consultado en http://www.conabio.gob.mx/insitucion/proyectos/resultados/ InfB002.pdf; el 15 de Noviembre de 2011.

Pino, J., E. Vázquez, F. Reid, y A. D. Cuarón. 2008. Sphiggurus mexicanus. En: UICN 2012. The IUCN Red List of Threatened Species. Version 2014.3. Consultado en www.iucnredlist. org el 2 de mayo de 2015.

ReID, A. F. 1997. A field guide to the mammals of Central America and Southeast Mexico. Oxford University Press. New York, E.E.U.U. 
Semarnat (Secretaría de Medio Ambiente y Recursos Naturales). 2010. NOM 059-SEMARNAT-2010, Protección ambiental-Especies nativas de México de flora y fauna silvestres-Categorías de riesgo y especificaciones para su inclusión, exclusión o cambio-Lista de especies en riesgo. Diario oficial, segunda sección, pags.1-78. Jueves 30 de Diciembre. Ciudad de México, México.

Voss, R. S. 2011. Revisionary notes on Neotropical porcupines (Rodentia: Erethizontidae) 3. An annotated checklist of the species of Coendou Lacépède, 1799. American Museum Novitates 3720:1-36.

Summited: April 13, 2015

Review: May 2, 2015

Accepted: August 3, 2015

Associated editor: Consuelo Lorenzo 